\title{
A Passive Approach for Detecting Image Splicing using Deep Learning and Haar Wavelet Transform
}

\author{
Eman I. Abd El-Latif \\ Mathematics Department, Faculty of Science, Benha University, Egypt. \\ E-mail: eman.mohamed@fsc.bu.edu.eg
}

\begin{abstract}
Ahmed Taha
Computer Science Department, Faculty of Computers and Informatics, Benha University, Egypt.

E-mail: ahmed.taha@fci.bu.edu.eg

Hala H. Zayed

Computer Science Department, Faculty of Computers and Informatics, Benha University, Egypt.

E-mail: hala.zayed@fci.bu.edu.eg
\end{abstract}

Received: 10 March 2019; Accepted: 20 March 2019; Published: 08 May 2019

\begin{abstract}
Passive image forgery detection has attracted many researchers in the recent years. Image manipulation becomes easier than before because of the fast development of digital image editing software. Image splicing is one of the most widespread methods for tampering images. Research on detection of image splicing still carries great challenges. In this paper, an algorithm based on deep learning approach and wavelet transform is proposed to detect the spliced image. In the deep learning approach, Convolution Neural Network (CNN) is employed to automatically extract features from the spliced image. CNN is applied and then Haar Wavelet Transform (HWT) is used. Support Vector Machine (SVM) is used later for classification. Additional experiments are performed. That is, Discrete Cosine Transform (DCT) replaces HWT and then Principle Component Analysis (PCA) is applied. The proposed algorithm is evaluated on a publicly available image splicing datasets (CASIA v1.0 and CASIA v2.0). It achieves high accuracy while using a relatively low dimension feature vector. Our results demonstrate that the proposed algorithm is effective and accomplishes better performance for detecting the spliced image.
\end{abstract}

Index Terms - Splicing Image Forgery, Tampered Image Detection, Convolution Neural Network (CNN), Haar Wavelet Transform (HWT), Discrete Cosine Transform (DCT), Support Vector Machine (SVM).

\section{INTRODUCTION}

Modern image processing tools and computer graphics software (such as Adobe Photoshop) allow anyone to modify the content of the digital image. It is easy to manipulate the digital images but, it is hard to confirm the integrity of digital images by naked eyes. Consequently, image forgery detection becomes a hot research field. In general, there are two techniques for digital image forensics. These techniques can be classified into two types: active techniques $[1,2]$ and passive techniques $[3$, 4]. Active techniques are based on inserting watermarks or signatures in digital images during recording. In this technique, the authentication of the digital image is checked by verifying if the extracted watermark matches the original one. The major limitation of the active technique is that it requires specially equipped cameras to insert the watermark during the digital image creation. In contrast, the passive techniques can authenticate an image without the need of the original image. One of the most popular types of image manipulation is image splicing. It manipulates images by cropping or copying a region from one image and pasting it into another image. Fig. 1 shows an example of image splicing. The spliced image is shown in Fig.1.c. It is created by merging two original images (Fig.1.a and 1.b).

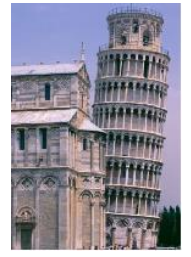

(a) original image 1

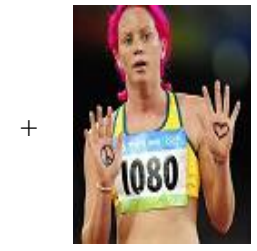

(b) original image 2

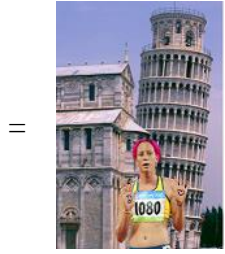

(c) spliced image
Fig.1. Example of Spliced Image

The forged images may be used for malicious purposes; this can lead to irreparable damage to human society. After studying existing algorithms towards detection of the spliced region, it was found that the existing algorithms achieve high dimensional feature vectors. In 
this paper, an algorithm is proposed to detect image splicing forgery. It employs a deep learning approach that is based on the use of the Convolutional Neural Network $(\mathrm{CNN})$. CNN is one of the commonly used models in deep learning [5]. It has many distinct characteristics that make it widely used. In $\mathrm{CNN}$, feature extraction and classification processes are generated at the same network. The weights of the convolutional layers are determined during the training process. In addition, CNN utilizes a less number of connections within the same network.

The rest of this paper is organized as follows. In Section 2, an overview of the related work is given. Section 3 presents the proposed algorithm for detecting image splicing forgery in details. The experimental results using different datasets are discussed in Section 4. Finally, the paper is accomplished in section 5.

\section{RELATED WORK}

In the recent years, several types of passive image splicing detection algorithms are suggested. These algorithms can be divided into three main types based on the technique used for features extraction. The three types are Local Binary Pattern (LBP), Markov model, and deep learning.

In the first type, LBP is utilized for extracting features from the tampered images [6, 7, 8]. Yujin et al. [6] suggest an algorithm for image splicing detection. The algorithm is based on Discrete Cosine Transform (DCT) and LBP. First, the color image is divided into multi-size blocks. Then, DCT is applied to each block. Later, LBP is applied for extracting features in each block. The final feature vector is formed by concatenating different LBP histograms. Moreover, Support Vector Machine (SVM) is employed to classify spliced and original images by utilizing the dimensionality-reduced features.

Ghulam et al. [7] use Steerable Pyramid Transform (SPT) and LBP to detect image splicing. In the first stage, the color image is converted into $\mathrm{YCbCr}$ chrominance space. Then, SPT is applied in each chrominance component in the second stage. Features are extracted using LBP from each SPT sub-band. Different LBP histograms are merged to form the final features vector. Finally, SVM is applied in the final step to check whether the color image is forged or not.

In [8], a forgery detection algorithm based on Discrete Wavelet Transform (DWT) and LBP is suggested. The color image in the algorithm is converted into $\mathrm{YCbCr}$ color space. For $\mathrm{Cr}$ chrominance channel, DWT is applied to get the low-level coefficients. Furthermore, features are extracted from the four sub-bands (LL, LH, $\mathrm{HL}, \mathrm{HH})$ using LBP. Histograms are concatenated from the four sub-bands to form the final feature vector. Then, SVM is used in the classification process based on the final feature vector.

In general, LBP suffers from some limitations. That is, it generates $2^{\mathrm{P}}$ features, where $\mathrm{P}$ is the number of neighbors. If $\mathrm{P}$ is increased, the numbers of features increase in turn. Another limitation of using LBP is that many different structural patterns may have the same
LBP code. Furthermore, it is very sensitive to noise.

Moreover, in the second type, Markov models are utilized widely for feature extraction [9, 10, 11]. In [9], an algorithm for passive detection of image splicing is presented. It extracts features from both spatial and frequency domains. In the spatial domain, the pixel value is subtracted from its neighboring pixel value in different directions. In the frequency domain, DCT is utilized and then the correlation is calculated between DCT coefficients and its neighbors. Also, Principal Component Analysis (PCA) is then applied to convert feature vectors into a lower dimensional space. Gaussian RBF kernel is used in SVM for classification spliced and original images.

In addition, an algorithm based on Markov and Quaternion discrete cosine transform (QDCT) is suggested for image splicing detection in [10]. The color image is blocked into three color components: Red, Green and Blue; and each block is still a color image. For each block, the QDCT transform is applied. Then, the horizontal, vertical, main diagonal and minor diagonal are calculated for each block. The histogram intersection in SVM is utilized for classification.

In [11], Markov feature extraction algorithm for splicing image detection is suggested. The algorithm is based on the maximum pixel value of the different direction in the DCT domain. These maximum pixels values are utilized to choose Markov features. In addition, a threshold expansion is utilized to reduce the information loss caused by the coefficient threshold. The increased number of features is reduced by using the even-odd Markov algorithm. However, Markov models suffer from the complexity of calculations and time consumption.

In the third type, image splicing detection algorithms using deep learning are suggested [12, 13, 14]. In [12], Ying et al. present a two-stage deep learning algorithm to extract the feature from tampered images. As a preprocessing step, the color image is converted into $\mathrm{YCrCb}$ color space. Then, the image is segmented into patches and for each patch; the 2D Daubechies wavelet transform is applied. A Stacked Auto-Encoder model (SAE) is utilized to extract feature in the first stage. In fact, SAE is a type of neural networks which contains three hidden layers and an MLP layer. In the second stage, the contextual information of each patch is integrated to give better accuracy. The limitation of this algorithm is that it contains a small number of hidden layers and hence it cannot extract effective features.

In [13], a universal forensic algorithm to detect manipulation in the image using deep learning is suggested. This algorithm utilizes CNN. The used CNN contains two convolutional layers, two max-pooling layers, and three fully connected layers. The number of features is bigger than the feature in the next algorithm.

An algorithm to extract the features form the spliced image using deep learning is presented [14]. The used CNN consists of six convolutional layers and three maxpooling layers. After the features are extracted from $\mathrm{CNN}$, PCA is utilized to reduce the dimension of features. The 
final features are utilized in SVM to classify the image.

\section{The Proposed ALGORITHM}

The main objective of our work is to improve image splicing detection using the deep learning approach. Fig. 2 shows the block diagram of the proposed algorithm to detect the image splicing. Actually, the deep learning is a kind of multi-layer neural networks, in which each layer utilizes the output from the previous layer as an input to the next layer. Many models of the deep learning are presented including Deep Belief Network [15], Deep Auto Encoder and Convolutional Neural Network (CNN) [16]. CNN is a type of neural network models, used to extract features automatically from the training data. In the proposed algorithm, CNN is used to extract features, and then HWT is applied. Finally, SVM is employed for classification.

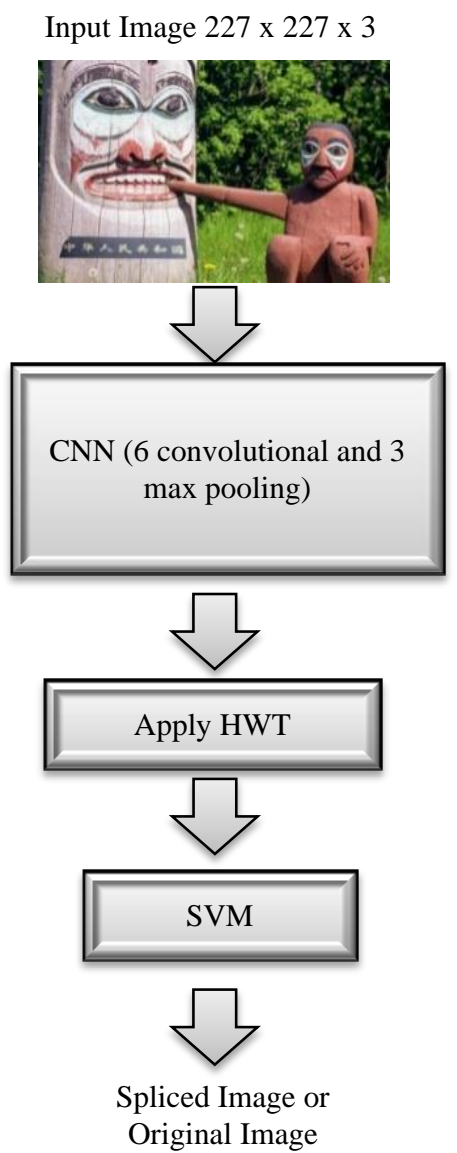

Fig.2. Block Diagram of the Proposed Algorithm

\section{A. CNN Architecture}

The architecture of CNN is a series of computational layers stacking together and finishing with a classifier. CNN contains a higher number of hidden layers which is different from the traditional network. CNN is a sparse network because it contains a less number of connections within the same network. Furthermore, one of the main advantages of $\mathrm{CNN}$ is the weight sharing. Hence, the network can work faster. However, in traditional neural networks, each element weight is utilized once in computing the output layer. In $\mathrm{CNN}$, the equivariant representation permits the network to detect edge, texture, and shape in different locations. Basic components of $\mathrm{CNN}$ are a convolution layer, non-linearity layer, and a pooling layer. The proposed CNN consists of six convolutional layers and three pooling layers as shown in Fig. 3. The input layer of $\mathrm{CNN}$ is $227 \times 227 \times 3(227 \times 227$ patch and 3 color channels). The output volume $\mathrm{W}_{2} \times \mathrm{H}_{2}$ $\times F$ is calculated by the equations [17]:

$$
\begin{aligned}
& W_{2}=\frac{W_{1}-F+2 P}{S}+1 \\
& H_{2}=\frac{H_{1}-F+2 P}{S}+1
\end{aligned}
$$

where $W_{1}$ is the width of the input volume, $H_{1}$ is the height of the input volume, and $r$ is the number of channels)

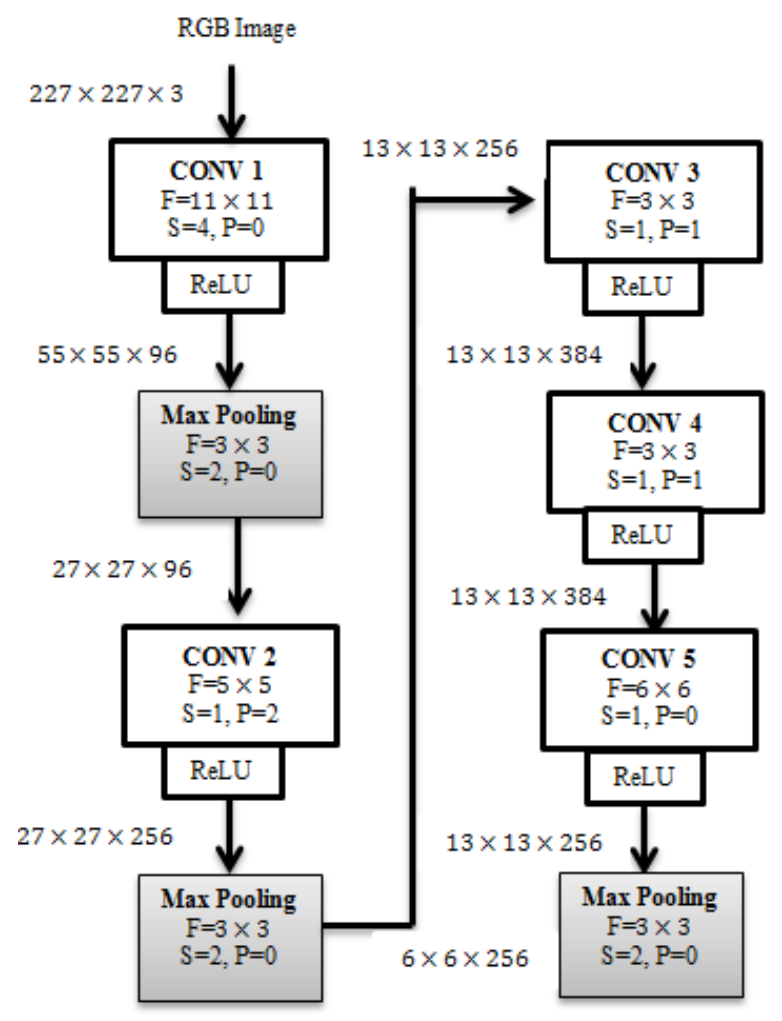

Fig.3. Architecture of the Proposed CNN

The convolution layer is the first layer in CNN. The parameters of convolution layers are the stride, the padding and the size of the filter. The stride controls how many positions the filter convolves around the input image. Common values of stride are $(1,1),(2,2)$, and (4, 4). Padding means inserting zeros on the borders of the image. In our algorithm, six convolution layers are applied. As shown in Figure 3, conv1 has 96 kernels with a filter size of $11 \times 11$. Conv 2 and conv5 have 256 kernels but the filter size in conv 2 is $5 \times 5$ and conv5 is $6 \times 6$. Conv 3 and Conv 4 have 384 kernels of size $3 \times 3$. The final convolution yields $1 \times 1 \times 4096$ feature maps with the kernel of size $6 \times 6, S=2$, and $P=0$. 
After each convolution layer, The Rectified Linear Unit (ReLU) is applied as an activation function [18]. The output is 0 or $\mathrm{x}$ (value of the pixel) depending on the sign of the pixel value. The output is 0 if the sign is negative, and the output is equal to $\mathrm{x}$ if the sign is positive. Compared to the common activation functions (tanh and sigmoid), ReLU is the better activation function. ReLU is the simplest for computation and the fastest in training big data [13].

After a few ReLU layers, a pooling layer is utilized. The pooling layer is applied to reduce the dimension of features from the convolution layer and decrease the computation complexity. There are two possible types of pooling layer: max pooling and average pooling. Max pooling is better than average pooling because it is very fast and efficient way in computation. As shown in Figure 3, three max pooling is employed in the proposed algorithm. Pooling layer uses a filter with size $3 \times 3$, stride 2 and pad 0 .

\section{B. Wavelet Transforms}

There are different types of wavelet transforms. Wavelet transforms translate the image from the spatial domain to the frequency domain [19]. There are different types of wavelet transforms but in the proposed algorithm Haar Wavelet Transform (HWT) is utilized. HWT is the simplest one and also, it has efficient memory and fast. After HWT is applied, a two-dimensional array is obtained. This array contains four bands categorized as LL (Low-Low), HL (High-Low), LH (Low-High), and $\mathrm{HH}$ (High-High). For example, if the sub-band image is created using a high filter on the rows and a low filter on the columns, it is called the HL sub-band. It is noted that HWT reduces features from 4,096 to 1,024.

\section{Principal Components Analysis}

PCA is one of the most common techniques that is widely used in data dimensionality reduction. It transforms the higher dimensional space that may cause a curse of dimensionality problem into lower dimensional space. PCA utilizes four steps to reduce the dimension of the feature. The first step is the image normalization but in the second step, the covariance matrix is calculated. Eigenvectors and the corresponding eigenvalues are computed in the third step. Finally, the original data is transformed into the new feature vector [20].

\section{EXPERIMENTAL RESULTS}

In order to measure the performance of the proposed algorithm, several experiments have been performed. The proposed CNN model is implemented using Matlab R2016b. It uses the Caffe deep learning framework. In the first subsection, the description of the datasets used for evaluating the proposed algorithm is provided. Evaluation metrics are presented in the next subsection. Finally, the results of the experiments are analyzed and discussed.

\section{A. Datasets Description}

Two public datasets are used to test the proposed algorithm: CASIA v1.0 [21] and CASIA v2.0 [22]. They are the most commonly used datasets for detecting spliced image $[6,7,8]$. The total number of images in CASIA v1.0 is 1721, and in CASIA v2.0 is 12,614. A detailed description of the two databases is given in Table 1 .

Table 1. Overview of CASIA v1.0 and CASIA v2.0

\begin{tabular}{|c|c|c|c|c|}
\hline Dataset & $\begin{array}{c}\text { Image } \\
\text { type }\end{array}$ & Image Size & Authentic & Spliced \\
\hline $\begin{array}{c}\text { CASIA } \\
\text { v1.0 }\end{array}$ & jpg & $384 \times 256$ & 800 & 921 \\
\hline $\begin{array}{c}\text { CASIA } \\
\text { v2.0 }\end{array}$ & $\begin{array}{c}\text { jpg } \\
\text { tif } \\
\text { bmp }\end{array}$ & $\begin{array}{c}240 \times 160 \\
\text { To } \\
900 \times 600\end{array}$ & 7491 & 5123 \\
\hline
\end{tabular}

Fig. 4 provides some example images of CASIAV1.0 Dataset. The first row is the original images and the second row is the forged images.

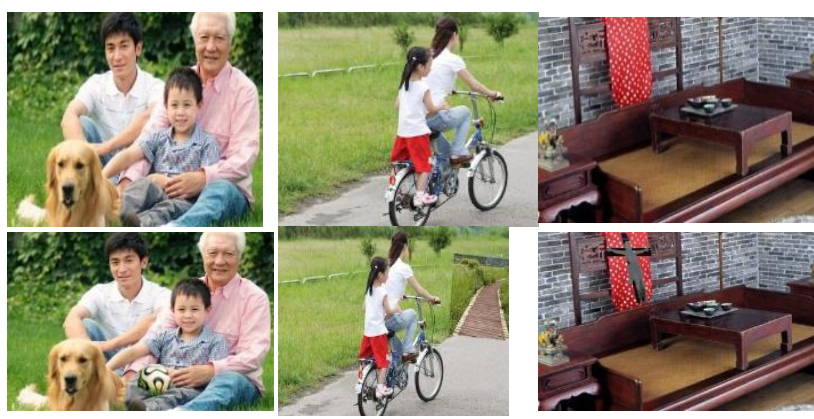

Fig.4. Some Example Images in CASIAV1.0 Dataset

\section{B. Evaluation Metrics}

To measure the performance of the proposed algorithm, several evaluation metrics are used [12, 13, 14] including accuracy, recall, precision, and F-measure metrics.

1. Accuracy is defined as the percent ratio of the images that are accurately classified and it is calculated by the following equation [23]:

$$
\text { Accuracy }=\frac{T P+T N}{T P+T N+F N+F P} \times 100
$$

where TP (True Positive) is defined as the number of spliced image labeled as tampered. FN (False Negative) is the number of spliced images labeled as an original. TN (True Negative) is the number of original images labeled as an original. FP (False Positive) is the number of original images labeled as tampered.

2. The recall is the proportion of all positive cases that are accurately classified separated by all True Positive cases [23]. It also is known as True Positive Rate (TPR) and it is calculated by the following equation [23]:

$$
\text { Recall }=\frac{T P}{T P+F N} \times 100
$$


3. Precision is known as positive predictive value and it is computed as [23]:

$$
\text { precision }=\frac{T P}{T P+F P} \times 100
$$

4. F- Measure is the harmonic mean of precision and recall and it is computed as [23]:

$$
F-\text { Measure }=2 * \frac{\text { Recall } * \text { Precision }}{\text { Recall }+ \text { Orecision }}
$$

\section{COMPARISON WITH OTHER PASSIVE ALGORITHMS}

In this section, the proposed algorithm is compared with some recent algorithms including: DWT + LBP [8], Markov features + QDCT [10], a deep learning [12], a grey level run length matrix (GLRLM) [24], and Markov feature [25]. All experiments are performed using the same image library (CASIA v1.0 and CASIA v2.0). In comparative experiments, not only the accuracy is considered, but also the feature dimension is taken into account.

Table 2. Experimental Results of the Proposed Algorithm and other Algorithms on CASIA v1.0

\begin{tabular}{|c|c|c|c|}
\hline Methods & Accuracy & Precision & Recall \\
\hline $\begin{array}{c}\text { Mandeep } \text { et al. } \\
(2016) \text { [8] }\end{array}$ & $92.62 \%$ & N/A & $89.25 \%$ \\
\hline $\begin{array}{c}\text { Ying } \text { et al. } \\
(2016)[12]\end{array}$ & $87.51 \%$ & $59.43 \%$ & N/A \\
\hline $\begin{array}{c}\text { Sahar } \text { et al. } \\
(2013) \text { [23] }\end{array}$ & $94.19 \%$ & N/A & N/A \\
\hline $\begin{array}{c}\text { Saba } \text { et al. } \\
(2014) \text { [24] }\end{array}$ & $80.71 \%$ & N/A & N/A \\
\hline $\begin{array}{c}\text { The Proposed } \\
\text { Algorithm }\end{array}$ & $\mathbf{9 4 . 5 5 \%}$ & $\mathbf{9 5 . 1 4 \%}$ & $\mathbf{9 8 . 9 9 \%}$ \\
\hline
\end{tabular}

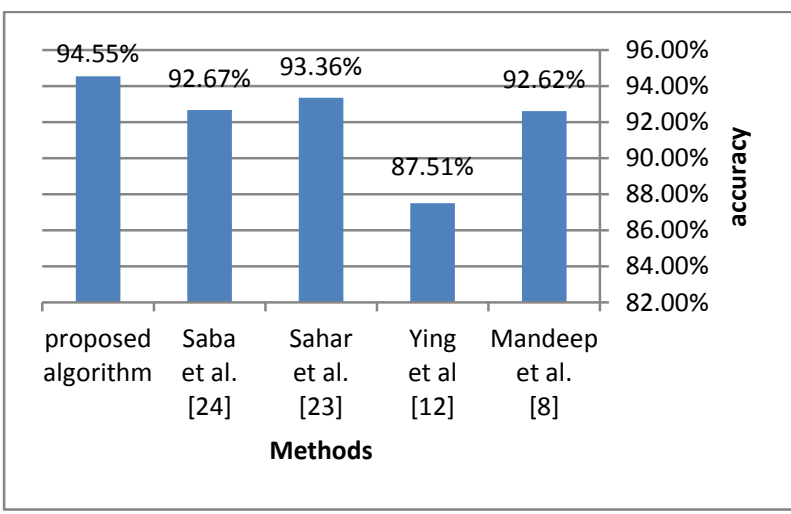

Fig.5. Accuracy Comparison of Proposed Algorithm with Existing Methods on CASIA v1.0

Table 2 shows the precision, recall, and accuracy results of different methods for image splicing forgery detection on CASIA v1.0. As it can be noticed, the proposed algorithm is better than the algorithm presented in [12] because it has less number of hidden layers. Moreover, the proposed algorithm outperforms the algorithm presented in [8] that uses LBP. In fact, LBP is sensitive to the noise as well as many different structural patterns may have the same LBP code. Fig.5. shows the comparison of accuracy between the proposed algorithm and other algorithms on CASIA v1.0. The proposed algorithm yield the best detection performance compared with other algorithms, achieving $94.55 \%$ accuracy on the full CASIA v1.0 dataset. Precision and recall are $95.14 \%$ and $98.99 \%$, respectively

In order to better demonstrate the potential of the proposed algorithm, extra experiments on CASIA v2.0 are conducted. Table 3 shows the precision, recall, and accuracy results of different methods for image splicing forgery detection on CASIA v2.0. The proposed algorithm achieves $94.55 \%$ accuracy on the full CASIA v2.0 dataset. Precision and recall are $95.14 \%$ and $98.99 \%$, respectively. Fig.6. shows the comparison of accuracy between the proposed algorithm and other algorithms on CASIA v2.0

Table 3. Experimental Results of the Proposed Algorithm and other Algorithms on CASIA v2.0

\begin{tabular}{|c|c|c|c|}
\hline Methods & Accuracy & Precision & Recall \\
\hline $\begin{array}{c}\text { DWT+LBP } \\
{[8]}\end{array}$ & $94.09 \%$ & N/A & $91.87 \%$ \\
\hline $\begin{array}{c}\text { Markov features } \\
+ \\
\text { QDCT [10] }\end{array}$ & $92.38 \%$ & N/A & N/A \\
\hline $\begin{array}{c}\text { Deep Learning } \\
{[12]}\end{array}$ & $87 \%$ & $80.65 \%$ & N/A \\
\hline $\begin{array}{c}\text { GLRLM Texture } \\
\text { features [24] }\end{array}$ & $87.6 \%$ & N/A & N/A \\
\hline $\begin{array}{c}\text { Markov feature } \\
{[25]}\end{array}$ & $93 \%$ & $\mathbf{9 7 . 1 4 \%}$ & $\mathbf{9 9 . 0 3 \%}$ \\
\hline $\begin{array}{c}\text { Proposed } \\
\text { Algorithm }\end{array}$ & $\mathbf{9 6 . 3 6 \%}$ & \multicolumn{2}{|c|}{} \\
\hline
\end{tabular}

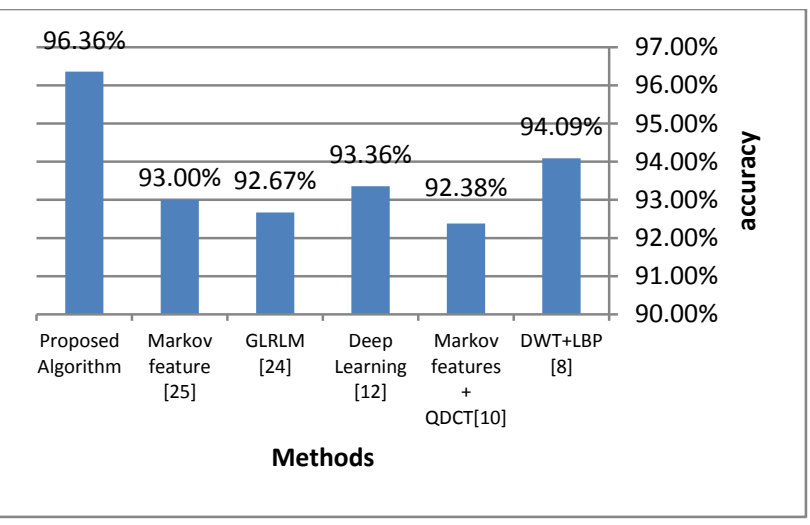

Fig.6. Accuracy Comparison of Proposed Algorithm with Existing Methods on CASIA v2.0

Table 4. Comparisons of Feature Vector Size

\begin{tabular}{|c|c|c|}
\hline Methods & Feature Vector Size & Accuracy \\
\hline $\begin{array}{c}\text { Ce Li } \text { et al. } \\
(2015)[10]\end{array}$ & 1,452 & $92.67 \%$ \\
\hline $\begin{array}{c}\text { Sahar } \text { et al. } \\
(2013)[23]\end{array}$ & 1,920 & $94.19 \%$ \\
\hline $\begin{array}{c}\text { Matthias } \text { et al. } \\
(2010)[26]\end{array}$ & 2,744 & $91.15 \%$ \\
\hline $\begin{array}{c}\text { Xudong } \text { et al. } \\
(2015) \text { [27] }\end{array}$ & 14,240 & $93.36 \%$ \\
\hline Proposed Algorithm & $\mathbf{1 , 0 2 4}$ & $\mathbf{9 6 . 3 6 \%}$ \\
\hline
\end{tabular}


The algorithm is also tested against the size of the feature vector. Table 4 and Fig. 7 show the comparative results of the proposed algorithm and its counterparts. As shown in the table, the dimensionality of features in our algorithm is 1,024 that is the least size with respect to all the other four detection algorithms.

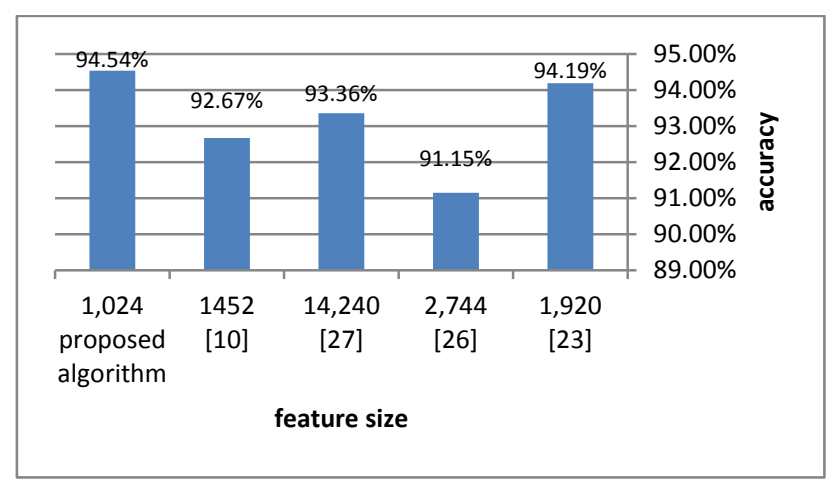

Fig.7. Comparisons of Feature Vector Size

\section{USING DCT INSTEAD OF HWT}

All the previous experiments have investigated the performance of the proposed algorithm in comparison with some other algorithms. However, for deeper analysis of the proposed algorithm, additional experiments are performed. That is, Discrete Cosine Transform (DCT) replaces HWT and then PCA is applied.

Table 5 shows the three performance metrics: accuracy, TPR, and precision of both the HWT-based algorithm and the DCT-based algorithm. It is noted that the HWT-based algorithm gives better detection accuracy than the DCTbased algorithm. It achieves accuracy equal to $94.55 \%$ for CASIA v1.0 and $96.36 \%$ for CASIA v2.0. However, DCT-based algorithm achieves a less accuracy for CASIA v1.0. The highest precision is achieved by HWTbased algorithm in CASIA v2.0. This is because DCT neglects the correlation between the pixels inside blocks and the pixels of the neighboring blocks.

Table 5. Accuracy, Recall, and Precision Comparison between HWTbased Algorithm and DCT-based Algorithm

\begin{tabular}{|c|c|c|c|c|}
\hline Datasets & \multicolumn{2}{|c|}{ CASIA v1.0 } & \multicolumn{2}{|c|}{ CASIA v2.0 } \\
\hline $\begin{array}{c}\text { Proposed } \\
\text { Algorithms }\end{array}$ & $\begin{array}{c}\text { CNN } \\
+ \\
\text { HWT }\end{array}$ & $\begin{array}{c}\mathrm{CNN} \\
+ \\
\mathrm{DCT}\end{array}$ & $\begin{array}{c}\text { CNN } \\
+ \\
\text { HWT }\end{array}$ & $\begin{array}{c}\text { CNN } \\
+ \\
\text { DCT }\end{array}$ \\
\hline Accuracy & $94.55 \%$ & $90.9 \%$ & $96.36 \%$ & $93.64 \%$ \\
\hline Recall & $95.14 \%$ & $93.2 \%$ & $97.14 \%$ & $95.19 \%$ \\
\hline Precision & $98.99 \%$ & $96.96 \%$ & $99.03 \%$ & $98 \%$ \\
\hline F-Measure & $97.03 \%$ & $95.04 \%$ & $98.08 \%$ & $96.57 \%$ \\
\hline
\end{tabular}

\section{CONCLUSION}

Image splicing is one of the famous techniques used for image forgery. The forger simply copies and pasts some parts from one image into another image. In this paper, an algorithm for detecting image-splicing forgery is presented. The proposed algorithm employs a deep learning approach along with HWT. CNN is utilized to automatically generate features from the color image. The proposed algorithm uses CNN plus HWT to generate the final features. Finally, SVM is used for classification. Several experiments were conducted to evaluate the performance of the proposed algorithm. Additional experiments are performed. That is, Discrete Cosine Transform (DCT) replaces HWT and then Principle Component Analysis (PCA) is applied. The proposed algorithm is tested on two standard tampered image datasets: CASIA v1.0 and CASIA v2.0. It outperforms the recent algorithms in terms of accuracy, precision, and TPR. Furthermore, it has a low dimensional feature vector. Future work should concentrate on enhancing the image forgery detection by locating where the forgery is in a spliced image.

\section{REFERENCES}

[1] Chandan Kumar, Amit Kumar Singh, and Pardeep Kumar,"A recent survey on image watermarking techniques and its application in e-governance," Multimedia Tools and Applications, Springer, Volume 77, Number 3, pp. 3597-3622, September 2017.

[2] A.S.Kapse, Sharayu Belokar, Yogita Gorde, Radha Rane, and Shrutika Yewtkar," Digital Image Security Using Digital Watermarking," International Research Journal of Engineering and Technology, Volume 5, Number 3, pp. 163-166, March 2018.

[3] Tanzeela Qazi, Khizar Hayat, Samee U. Khan, Sajjad A. Madani, Imran A. Khan, Joanna Kołodziej, Hongxiang Li, Weiyao Lin, Kin Choong Yow, and Cheng-Zhong $\mathrm{Xu}$, "Survey on Blind Image Forgery Detection," In the Journal of IET Image Processing, Volume 7, Number 7, pp. 660-670, October 2013.

[4] Hany Farid, "A Survey of Image Forgery Detection," In the Journal of IEEE Signal Processing, volume 26,Number 2, pp. 16-25, March 2009.

[5] Weibo Liua, Zidong Wanga, Xiaohui Liua, Nianyin Zengb, Yurong Liucd, and Fuad E.Alsaadid," A survey of deep neural network architectures and their applications," Neurocomputing, ELSEVEIR, Volume 234, Number 19 ,Pages 11-26, April 2017.

[6] Yujin Zhang, Chenglin Zhao, Yiming Pi, Shenghong Li, and Shilin Wang," Image - splicing forgery detection based on local binary patterns of DCT coefficients. Security and Communication Networks," In the Journal of Security and Communication Networks, Volume 8, Number 14, pp. 2386-2395, September 2015.

[7] Ghulam Muhammad, Munner H. Al-Hammadi, Muhammad Hussain, and George Bebis, "Image Forgery Detection using Steerable Pyramid Transform and Local Binary Pattern," In the Journal of Machine Vision and Applications, Volume 25, Number 4, pp. 985-995, May 2014.

[8] Mandeep Kaur, and Savita Gupta, "A Passive Blind Approach for Image Splicing Detection Based on DWT and LBP Histograms," In the Proceedings of International Symposium on Security in Computing and Communication, Chandigarh, India, pp. 318-327, September 2016.

[9] E-Sayed M. El-Alfy, and Muhammad Ali Qureshi, "Combining spatial and DCT based Markov features for enhanced blind detection of image splicing," In 
International Journal of Pattern Analysis and Applications, Volume 18, Number 3, pp. 713-723, August 2015.

[10] CeLi, QiangMa, LimeiXiao, MingLi, and AihuaZhang," Image splicing detection based on Markov features in QDCT domain," In International Journal of Neurocomputing in ELSEVIER, Volume 228, Number 8, pp. Pages 29-36, March 2017.

[11] Jong Goo Han, Tae Hee Park, Yong Ho Moon, and Il Kyu Eoma," Efficient Markov feature extraction method for image splicing detection using maximization and threshold," In International Journal of Electronic Imaging, Volume 25, Number 2, April 2016.

[12] Ying Zhang, Jonathan Goh, Lei Lei Win, and Vrizlynn LL Thing, "Image Region Forgery Detection: A Deep Learning Approach," In the Proceedings of the Singapore Cyber-Security Conference (SG-CRC), pp. 111, January 2016.

[13] Belhassen Bayar, and Matthew C. Stamm, "A Deep Learning Approach to Universal Image Manipulation Detection using a New Convolutional Layer," In the proceedings of the 4th ACM Workshop on Information Hiding and Multimedia Security, Vigo, Galicia, Spain, pp. 5-10, June 2016.

[14] Eman I. Abd El-Latif, Ahmed Taha, and Hala H. Zayed," A Deep Learning Approach for Detecting Splicing Forgeries in Images," International Journal of Computer Science and Information Security, Vol. 16 No. 10, 2018.

[15] Honglak Lee, Chaitanya Ekanadham, and Andrew Y. $\mathrm{Ng}$. "Sparse deep belief net model for visual area V2," In the proceedings of neural information processing systems, Vancouver, British Columbia, Canada, pp. 873-880, December 2008

[16] Hugo Larochelle, Yoshua Bengio, Jérôme Louradour, and Pascal Lamblin, "Exploring strategies for training deep neural networks," In the Journal of Machine Learning Research, volume 10, pp. 1-40, January 2009.

[17] Aker Cemal, and Sinan Kalkan, "Using Deep Networks for Drone Detection," Advanced Video and Signal Based Surveillance (AVSS),In the proceeding of 14th IEEE International Conference on Advanced Video and Signal based Surveillance, Lecce, Italy, pp. 1-6, August 2017.

[18] Alex Krizhevsky, Ilya Sutskever, and and Geoffrey E. Hinton, "ImageNet Classification with Deep Convolutional Neural Networks," In the proceedings of Advances in neural information, USA, pp. 1097-1105, December 2012.

[19] Nick Kingsbury and Julian Magarey," Wavelet Transforms in Image Processing," In the proceedings of Signal analysis and prediction, Birkhäuser, Boston, pp. 27-46, March 1998.

[20] Lifei Zhan, Yuesheng Zhu, and Zhiwei Mo, "An Image Splicing Detection Method Based on PCA Minimum Eigenvalues," In the Journal of Information Hiding and Multimedia Signal Processing, Volume 7, Number 3, May 2016.

[21] CASIA Tampered Image Detection Evaluation Database (CASIA TIDE v1.0)" Available at: http://forensics.idealtest.org:8080/index_v1.html.

[22] CASIA Tampered Image Detection Evaluation Database (CASIA TIDE v2.0)" Available at: http://forensics.idealtest.org:8080/index_v2.html.

[23] Sahar Q Saleh, Muhammad Hussain, Ghulam
Muhammad, and George Bebis," Evaluation of Image Forgery Detection using Multi-scale Weber Local Descriptors," In the Journal of International Symposium on Visual Computing, Volume 24, Number 4, pp. 416424, August 2015.

[24] Saba Mushtaq, and Ajaz Hussain Mir," Novel method for image splicing detection," In the proceedings of Advances in Computing, Communications and Informatics (ICACCI), New Delhi, India, pp. 24-27, September 2014.

[25] Zhongwei He, Wei Lu , Wei Sun , and Jiwu Huang, "Digital image splicing detection based on Markov features in DCT and DWT domain," In the Journal of Pattern Recognition, Volume 45, Nunber 12, pp. 42924299, December 2012.

[26] Matthias Kirchner and Jessica Fridrich, "On detection of median filtering in digital images," In the proceedings of SPIE, Media Forensics Secure. II, volume 7541, pp. 754110-1-754110-12, January 2010.

[27] Xudong Zhao, Shilin Wang, Shenghong Li, and Jianhua $\mathrm{Li}$, "Passive Image-Splicing Detection by a 2-D Noncausal Markov Mode," In the Journal of IIEEE Transactions On Circuits And Systems For Video Technology, Volume. 25, Number 2, February 2015.

\section{Authors' Profiles}

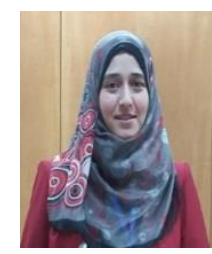

Eman I. Abd El-Latif received the M.Sc. degree in computer science, at Faculty of Science, Benha University, Egypt, in 2016. She is currently works as assistant lecturer at mathematics department, Benha University, Egypt. Her areas of research include Digital Forensics, Security (Encryption Steganography), and image processing.

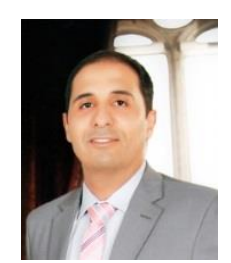

Dr. Ahmed Taha received his M.Sc. degree and his Ph.D. degree in computer science, at Ain Shams University, Egypt, in February 2009 and July 2015 respectively. $\mathrm{He}$ is currently works as assistant professor at computer science department, Benha University, Egypt. His research interests concern: Computer Vision \& Image Processing (Human Behavior Analysis - Video Surveillance Systems), Digital Forensics (Image Forgery Detection - Document Forgery Detection), Security (Encryption - Steganography - Cloud Computing), Content-Based Retrieval (Arabic Text Retrieval Video Scenes Classification-Video Scenes Retrieval Trademark Image Retrieval - Closed-Caption Technology).

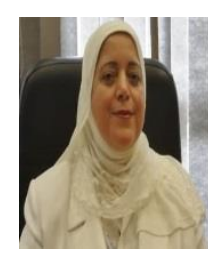

Prof. Hala H. Zayed received the B.Sc. in electrical engineering (with honor degree) in 1985, the M.Sc. in 1989 and Ph.D. in 1995 from Benha university in electronics engineering. She is now a professor at faculty of computers and informatics, Benha university. Her areas of research are computer vision, biometrics, machine learning and image processing. 
How to cite this paper: Eman I. Abd El-Latif, Ahmed Taha, Hala H. Zayed,"A Passive Approach for Detecting Image Splicing using Deep Learning and Haar Wavelet Transform", International Journal of Computer Network and Information Security(IJCNIS), Vol.11, No.5, pp.28-35, 2019.DOI: 10.5815/ijcnis.2019.05.04 